\title{
Beam damage assessment using natural frequency shift and machine learning
}

\author{
Nicoleta Gillich ${ }^{1}$, Cristian Tufisi 1 *, Christian Sacarea ${ }^{2}$, Catalin V. Rusu ${ }^{2}$, Gilbert-Rainer Gillich 1,3 , Zeno-Iosif \\ Praisach ${ }^{1,3}$ and Mario Ardeljan ${ }^{3}$
}

1 Department of Engineering Science, Babeș-Bolyai University, Str. M. Kogălniceanu 1, 400084 Cluj-Napoca, Romania; nicoleta.gillich@ubbcluj.ro (NG); cristian.tufisi@ubbcluj.ro (CT); gilbert.gillich@ubbcluj.ro (GRG); zeno.praisach@ubbcluj.ro (ZIP);

2 Department of Computer Science, Institute of German Studies, Babeș-Bolyai University, Str. M. Kogălniceanu 1, 400084 Cluj-Napoca, Romania; christian.sacarea@ubbcluj.ro (CS); vasile.rusu@ubbcluj.ro (CR).

3 Doctoral School of Engineering, Babeș-Bolyai University, Str. M. Kogălniceanu 1, 400084 Cluj-Napoca, Romania; mario.ardeljan@unbbcluj.ro (MA)

* Correspondence: cristian.tufisi@ubbcluj.ro (CT); gilbert.gillich@ubbcluj.ro (GRG)

\begin{abstract}
Damage detection based on modal parameter changes becomes popular in the last decades. Nowadays are available robust and reliable mathematical relations to predict the natural frequency changes if damage parameters are known. Using these relations, it is possible to create databases containing a large variety of damage scenarios. Damage can be thus assessed by applying an inverse method. The problem is the complexity of the database, especially for structures with more cracks. In this paper, we propose two machine learning methods, namely the random forest (RF) and the artificial neural network (ANN) as search tools. The databases we developed contain damage scenarios for a prismatic cantilever beam with one crack and ideal and non-ideal boundary conditions. The crack assessment is made in two steps. First, a coarse damage location is found from the networks trained for scenarios comprising the whole beam. Afterward, the assessment is made involving a particular network trained for the segment of the beam on which the crack is previously found. Using the two machine learning methods, we succeed to estimate the crack location and severity with high accuracy for both simulation and laboratory experiments. Regarding the location of the crack, which is the main goal of the practitioners, the errors are less than $0.6 \%$. Based on these achievements, we concluded that the damage assessment we propose, in conjunction with the machine learning methods, is robust and reliable.
\end{abstract}

Keywords: damage detection; linear regression; random forest; artificial neural network; training parameters; natural frequency

\section{Introduction}

Nondestructive damage detection methods have received increasing attention in recent decades and became a central research topic for scholars and practitioners belonging to the structural health monitoring community. Principles of vibration-based techniques, which are nowadays very popular, can be found in [1-3]. These methods are based on the deterministic relation between the damage characteristics (mainly location and severity), and the changes in the modal parameters (natural frequencies, mode shapes, and curvatures). Among the modal parameters, the natural frequencies are the easiest to determine and require the involvement of relatively cheap and very robust instrumentation. Moreover, estimating the natural frequencies require a limited number of sensors compared to methods based on mode shapes [4]. For this reason, in this paper, we focus on the analysis of natural frequencies to detect damage.

The temperature change can affect the natural frequencies of the structure. For accurate damage detection, it is important to remove the effects of temperature on the 
natural frequencies. An algorithm to this aim is presented in [5], where a successful damage assessment is performed for the case of changing temperatures. Mass changes also affect the natural frequencies of structures. In [6], it is investigated an analytical approach developed for a mass-spring-damper system that demonstrates how to localize mass change.

Detection and measurement of damage features for different crack types in slender beams using modal analysis is studied in [7]. Assessment of L and T-shaped cracks as well as delamination in bi-metal structures using natural frequencies is successfully performed in [8]. Assessment of corrosion and the analysis of the structural capacity of corroded I girders that belong to steel bridges is studied in [9].

In some real cases, multiple damages can occur at once, and the structures can have multiple supports. A complex study devoted to calculating the natural frequencies and mode shapes of multi-span beams is found in [10], and in [11] is presented a method to assess cracks in continuous beams. A study devoted to identifying multiple damages of multi-span bridges based on influence lines is presented in [12].

The complexity of damage (the crack shape and the orientation, the associated loss of mass, the number of cracks), as well as the complexity of the structures (multisupported beams, skeletal structures, the use of nonisotropic materials), increase the dimension of the dataset to be processed for damage detection. For this reason, researchers who develop methods for detecting damages are increasingly using Artificial Intelligence (AI) to analyze large amounts of data.

The Artificial Neural Network (ANN) is a commonly applied technique for SHM. A method to identify the damage location and its severity in a ten-floor structure, employing an auto-associative neural network combined with transmissibility is proposed in [13]. The development of a method to detect damages in a truss structure using an ANN is the subject of the research presented in [14]. A method to predict crack width for thick as well as for thin concrete elements, which bases on the feed-forward backpropagation and the radial basis neural networks, is proposed in [15].

The use of Random Forests (RF) and data fusion for structural damage detection are proposed in [16]. More recently, an RF model was used to Predict the Location of Potential Damage on Asphalt Pavement [17]. Here, RF data mining is used to analyze the interrelationships of variables. Another approach is found in [18]. The damage-sensitive features are extracted from raw sensor data using the cross-correlation function and wavelet packet decomposition. RF and other Ensemble Learning algorithms such as XGBoost are used to train the damage pattern classifier.

Despite successful assessment of damage, it is worth mentioning that the damage considered in most studies presented in the literature either manifests on an element with significant extent along the beam [13] or has significant severity: $22 \%$ to $68 \%$ in [19] and $20 \%$ to $80 \%$ in [20]. For skeletal structures, the damage manifests on one or more structural elements, see for example [14] and [16-18].

In prior research, we deduced a relation to calculate the natural frequencies of beams with known crack parameters. This relation is applicable also for beams with non-ideal boundary conditions. Being an analytical relation, it permits creating, easily and rapidly, a database with patterns for a multitude of damage scenarios. The database contains the Relative Frequency Shits (RFS) for eight out-of-plain vibration modes for all given damage locations, severities, and fixing conditions. It is used to train RF and ANN, with a huge amount of data. The training is first performed for scenarios covering all locations along the beam and afterward for locations on a specific segment. The proposed damage detection methodology presumes an initial/coarse assessment to find the crack location, followed by a second/fine assessment targeting the accurate crack location and severity.

To evaluate the effectiveness of the proposed approach, two examples, comprising numerical simulations and laboratory experiments on steel beams are performed. The results obtained by involving RF and ANN are compared; both methods lead to the correct location and quantification of the damage, regardless of the changes in the fixing conditions. It was possible to detect cracks with a much smaller depth than those reported 
in the literature. As far as we know, there are no methods for detecting defects that are applicable even in the event of a change in the conditions for fixing the beam.

The paper is organized as follows. After an introductory section, we present the theoretical background which permits developing a database consisting of an INPUT and a TARGET part. The methodology of RF and ANN, along with the training process based on the database are introduced in Section 3. In the next two sections, we prove the efficiency of the proposed approach. In section 4 we describe the numerical simulations performed on a steel cantilever beam, done to find the natural frequency shifts due to several damage cases. Then, we apply the two machine learning methods. In Section 5 we repeat the approach for laboratory-scale beams. Eventually, conclusions are formulated.

\section{Creating the database for Machine Learning (ML)}

As a crack propagates in a structure, it produces an alteration of structural stiffness. This alteration produces a change in modal parameters, of which the most obvious and easy to measure are changes in natural frequencies. There is a deterministic relationship between stiffness change and the decrease in the natural frequencies of the structure, therefore the position and depth of the crack can be identified if the changes in the frequencies produced by the defect are known. We proposed a robust and easy-to-use mathematical relation to predicting the natural frequency $f_{i-D}(x, a)$ of a cracked beam [21]. This relation relies on the features of the healthy beam, namely, the natural frequencies $f_{i-U}$ and the modal curvatures $\phi_{i}^{\prime \prime}(x)$ of the out-of-plain vibration modes, and the damage severity $\gamma(a)$, respectively. The mathematical relation is

$$
f_{i-D}(x, a)=f_{i-U}\left\{1-\gamma(a)\left[\bar{\phi}_{i}^{\prime \prime}(x)\right]^{2}\right\} .
$$

In this relation, we denoted the crack position with $x$, the crack depth with $a$, and $i$ stays for the mode number. From equation (1) we can deduce the relative frequency shift (RFS), which is the normalized frequency drop due to a crack, as

$$
\Delta \bar{f}_{i-D}(x, a)=\frac{f_{i-U}-f_{i-D}(x, a)}{f_{i-U}}=\gamma(a)\left[\bar{\phi}_{i}^{\prime \prime}(x)\right]^{2} .
$$

We find the two terms in the right part of equation (2) as follows. The crack severity is calculated involving an energy method, which says that a beam with a crack will be able to store less energy than a similar healthy beam and will suffer a greater deformation if subjected to a force [22]. So, we can calculate the severity involving the mathematical relation [23]

$$
\gamma(a)=\frac{\sqrt{\delta_{D}(a)}-\sqrt{\delta_{U}}}{\sqrt{\delta_{D}(a)}} .
$$

In this relation, we denoted $\delta_{u}$ the deflection of the healthy beam, and with $\delta_{D}(a)$ the defection of the beam with a crack that has depth $a$. Note that, the severity is calculated for the crack located at the position where the biggest curvature (or bending moment, which is proportional to the curvature) is achieved. In consequence, the position of the damage for which the severity is calculated differs depending on the boundary conditions of the beam; for example, for the cantilever beam that is the subject of the study presented hereinafter this position is the fixed end. On the other hand, the severity depends solely on the crack depth $a$, so that for a given depth it is the same regardless of the boundary conditions.

The effect of the crack position is controlled by the local curvature (or bending moment). This happens because the stress present in the affected slice is proportional to the bending moment. In consequence, a smaller effect on the frequency drop is obtained if the crack is not located at the particular position where the biggest bending moment is present. There are even situations in which the crack does not cause a decrease in 
frequency, namely when it is located at an inflection point [24]. To consider the effect of the crack position $x$ on the frequency drop, we use the normalized squared curvature. By normalization, we assign value one to the curvature or bending moment for the location where the beam is the most requested and a subunit value for the rest of the positions. For the cantilever beam, the normalized curvature is

$$
\bar{\phi}_{i}^{\prime \prime}(x)=0.5\left\{\cos \left(\lambda_{i} x\right)+\cosh \left(\lambda_{i} x\right)-\frac{\cos \lambda_{i}+\cosh \lambda_{i}}{\sin \lambda_{i}+\sinh \lambda_{i}}\left[\sin \left(\lambda_{i} x\right)+\sinh \left(\lambda_{i} x\right)\right]\right\} .
$$

where we denoted with $\lambda_{i}$ the eigenvalue of the $i$-th vibration mode. These values are indicated in the literature for different boundary conditions of the beam. Note that, other boundary conditions also lead to other curvature functions.

Taking into account the above, we can summarize that:

- The severity $\gamma(a)$ depends on the crack depth $a$ and is independent of the crack position $x$, boundary conditions, and vibration mode number $i$. Therefore, the severity for a beam with a given crack once calculated using equation (3) is valid for that beam irrespective of the boundary conditions. In practice, we calculate the severity using static finite element analysis for a cantilever beam because it presents an important deflection at the free end. A comprehensive description of the procedure to determine the correct severity is given in [25].

- $\quad$ The value of the normalized modal curvature $\bar{\phi}_{i}^{\prime \prime}(x)$ at the position $x$ where the crack is located reduces the effect of the severity since at that position less stress is stored in the beam. This term depends on the vibration mode number $i$ and the boundary conditions. Therefore, equation (2) is has a large degree of generality; it can be properly used for any support type if the correct curvature function $\bar{\phi}_{i}^{\prime \prime}(x)$ is employed. Hereinafter we exemplify the case of a cantilever, thus equation (4) is used.

In Figure 1 we represent the RFS calculated using equation (2) for vibration modes 1 and 3, for two crack depths.

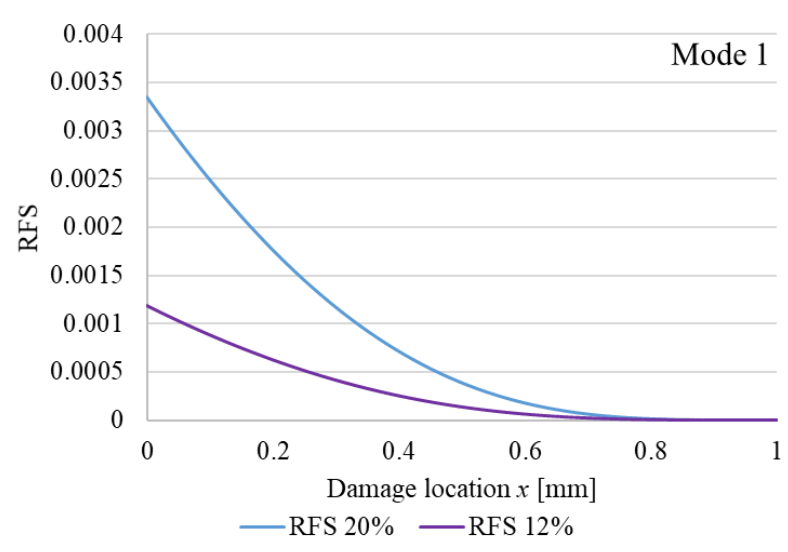

(a)

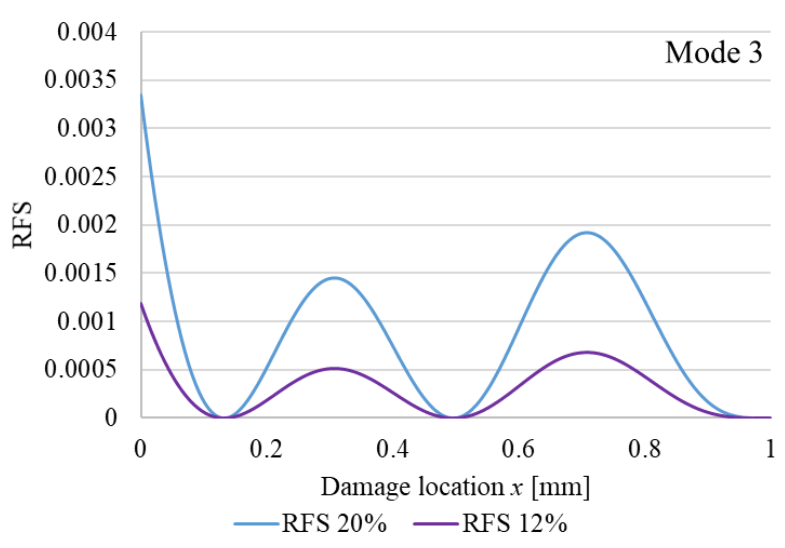

(b)

Figure 1. Plotted RFS functions for out-of-plain bending vibrations of a cantilever beam with perfect fixing: (a) vibration mode 1 ; (b) vibration mode 3 . The functions are plotted for a cross-section reduction of $12 \%$ and $20 \%$, respectively.

The above approach is valid for perfect boundary conditions. If the fixing is nonideal, the frequency drop is bigger. In prior research, we demonstrated that the superposition principle is valid [26]. For a beam with two cracks, which have position $x_{1}$ and $x_{2}$, and depths $a_{1}$ and $a_{2}$, respectively, the frequency drop is calculated as the sum of the two RFS, that is 


$$
\Delta \bar{f}_{i-D}\left(x_{1}, a_{1}, x_{2}, a_{2}\right)=\gamma_{1}\left(a_{1}\right)\left[\bar{\phi}_{i}^{\prime \prime}\left(x_{1}\right)\right]^{2}+\gamma_{2}\left(a_{2}\right)\left[\bar{\phi}_{i}^{\prime \prime}\left(x_{2}\right)\right]^{2} .
$$

We simulate the non-ideal boundary condition, namely the weak fixing, as a crack at the clamped end. So, an additional rotation is possible [27], which leads to a frequency decrease. Knowing that the normalized modal curvature at the fixed end of the cantilever is $\bar{\phi}_{i}^{\prime \prime}(0)=1$, equation (5) becomes

$$
\Delta \bar{f}_{i-D}\left(0, a_{1}, x_{2}, a_{2}\right)=\gamma_{1}\left(a_{1}\right)+\gamma_{2}\left(a_{2}\right)\left[\bar{\phi}_{i}^{\prime \prime}\left(x_{2}\right)\right]^{2} .
$$

Hence, we can plot the RFS for the beam with non-ideal boundary conditions as shown in Figure 2.

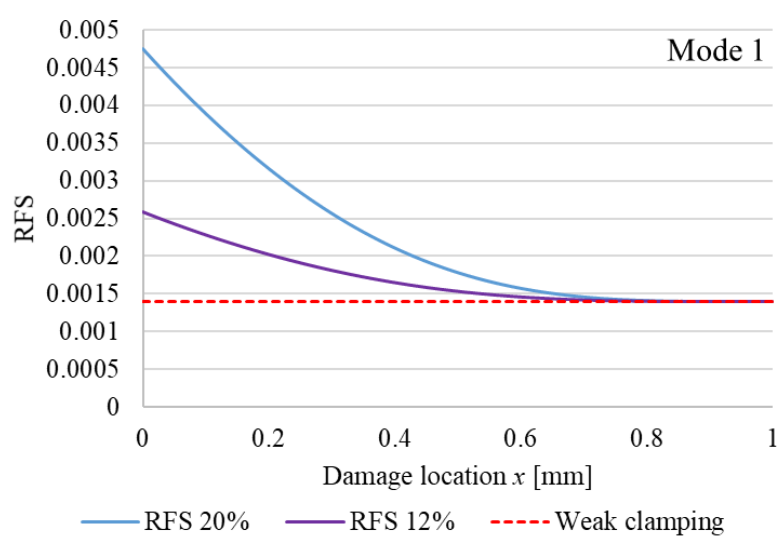

(a)

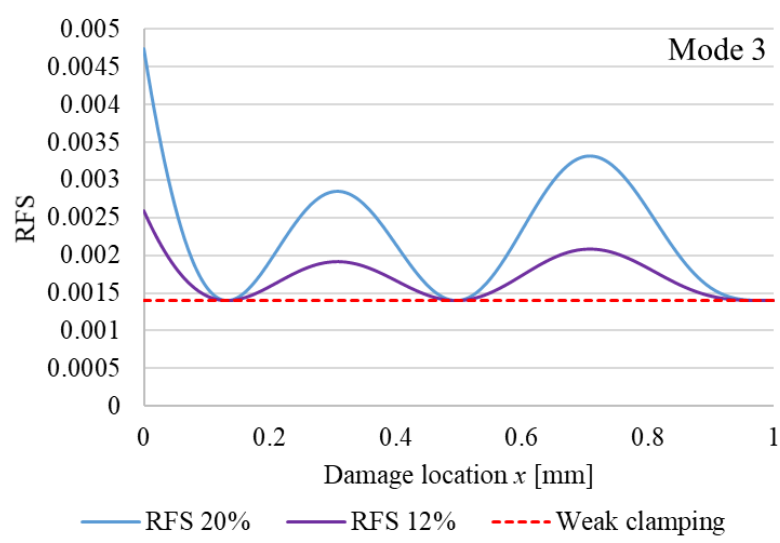

(b)

Figure 2. Plotted RFS functions for out-of-plain bending vibrations for a cantilever beam with non-ideal fixing: (a) vibration mode $1 ;(\mathbf{b})$ vibration mode 3 . The functions are plotted for a cross-section reduction of $12 \%$, respective $20 \%$.

The inverse method we developed to detect cracks implies, as a first step, calculating the RFS for numerous damage scenarios and creating a database. The database thus contains two distinct sections:

- TARGET elements, which are the local value of the curvature for a given position, the severity of the defect, and the severity corresponding to the weak fixing. These are placed on columns, the number of columns $m$ corresponding to the desired number of scenarios. The index of the column is denoted $k$, thus $k=1, \ldots, m$.

- INPUT elements, which are calculated relative frequency shifts $\mathrm{RFS}_{\mathrm{i}-\mathrm{k}}^{\mathrm{c}}$ for a chosen number of vibration modes $n$. These are also arranged in columns, each INPUT column corresponding to a TARGET column.

The second step is monitoring the structure, which presumes to measure the natural frequencies. If changes are observed, the frequency shifts calculated for the measured frequencies $\left(\mathrm{RFS}_{\mathrm{i}}^{\mathrm{m}}\right)$ are compared with the elements of the INPUT section of the database, i.e. RFS $_{i-k}^{c}$. The column number $k$ for which the best fit is obtained between the calculated and measured RFS indicates the crack position and severity. In this study, we propose a machine learning approach to find the best fit. The chosen methods are the random forest and the neural network.

\section{Machine Learning methods}

In the last decade, artificial intelligence (AI) has become a frequently used term for applications that perform complex tasks that once required human intervention, such as Structural Health Monitoring methods. In this section, we present the development and testing of two machine algorithms used for damage detection. The training data is generated using the method described in the previous chapter. The database contains 
36573 damage scenarios (number of columns in the INPUT and TARGET files). The number of rows in the INPUT file is eight, comprising the first eight out-of-plain bending vibration modes. The TARGET file consists of three elements, i.e. three rows, which are: the damage location, the damage severity, and the severity of the damage simulating the weak clamping. For this study, we considered 100 locations of the crack evenly distributed along the beam, the cracks having 17 levels of depth. The beam has both ideal and nonideal clamping, for the latter situation 7 cases of weak fixing are generated. It resulted in 36573 damage scenarios. The two files of the database are presented in [28].

Because of the complexity of the application and the accuracy required for determining the exact position and severity of transverse cracks we propose a two-step approach. This means that after a coarse localization of the crack using a network trained for all damage scenarios, we apply a second check, this time for a model trained for the specific section of the beam on which the crack is found at the first step. As an example, if the crack is found involving one of the developed methods at $175 \mathrm{~mm}$ from the fixed end, we make the second check for a segment extended between 100 and $250 \mathrm{~mm}$. For this segment, we train again the model for a limited number of inputs, considering just those which contain the crack position between the limits 100 and $250 \mathrm{~mm}$. This facilitates obtaining a better model of the structure and, in consequence, more precise localization of the crack.

To have a fast estimation, we divide the beam into nine segments, partially overlapped, and train the ML models a priori for these segments. Overlapping is used to avoid uncertainty regarding the choice of the right segment. The segments we used and the name of the network are presented in Table 1.

Table 1. Segments used to train the machine learning models for enhanced damage detection.

\begin{tabular}{lccccccccc}
\hline Segment limits & $0-150$ & $100-300$ & $250-400$ & $350-500$ & $450-600$ & $550-700$ & $650-800$ & $750-900$ & $850-1000$ \\
Network name & Sector 1 & Sector 2 & Sector 3 & Sector 4 & Sector 5 & Sector 6 & Sector 7 & Sector 8 & Sector 9 \\
\hline
\end{tabular}

The two ML methods and the settings applied for the training are presented in the next two sub-sections. For both methods, we use $70 \%$ of the data for training, $15 \%$ for testing, and $15 \%$ for validation.

\subsection{Random Forest}

Decision Trees have proven successful at exploring non-linear relationships between input and target variables [29]. Such trees work by splitting the dataset in instances that have a minimum amount of node impurity or in other words, are homogenous. Purity here means that each leaf node represents data points that are in the same class and is defined as the sum of square deviations in class predictions. The biggest drawback of decision trees is that they can easily overfit. This can be mitigated by aggregating such trees to reduce variance.

$\mathrm{RF}$ is a technique that employs an ensemble of decision trees and can be used for regression and classifications tasks. The prediction made by an RF aggregates the output of individual trees into a single variable [30]. When building trees, the algorithm randomly selects a given number of features. This essentially prevents multiple decision trees that rely on the same feature. The process is repeated until a group of regression trees, each trained on a randomly selected subset of data, is created. This induced randomness is what compensates for the weakness of each individual tree.

The performance of an RF model can be tweaked by tuning a few key parameters, with some studies reporting that there is a significant benefit to tuning RF parameters away from their default settings [31]. Table 2 summarizes the most common hyperparameters of the RF model. 
Table 2. RF Hyperparameters.

\begin{tabular}{ccc}
\hline Parameter & Meaning & Value \\
\hline n_estimations & The number of estimators in the forest & 400 \\
max_features & max number of features considered for splitting a node \\
max_depth & max number of levels in each decision tree & sqrt \\
min_samples_split & min number of data points placed in a node before the \\
node is split & 2 \\
min_samples_leaf & $\begin{array}{c}\text { min number of data points allowed in a leaf node } \\
\text { method for sampling data points. True= bootstrap } \\
\text { bootstrap }\end{array}$ & 1 \\
\hline
\end{tabular}

The number of trees in the forest should be in principle as high as possible but in practice, performance plateaus appear after a few hundred trees. In general, increasing the number of featur considered in splitting a node will improve performance as each node will have now have a higher number of options to consider, a lower value will increase the chance of selecting features with small effects which in turn could lead to improved performance in cases where such feature would be masked. Parameter min_samples_leaf represents the minimum size of terminal nodes. A higher number will lead to small trees while a smaller leaf size makes the tree more prone to noise in data.

The hyperparameters of the RF were tuned using a randomized search (RandomizedSearchCV in Scikit-Learn) with 5-fold cross-validation, that randomly chooses one of the possible values for each one of the hyperparameters and scores the estimator. The best estimator is used in the model. Table 2 also shows the tuned values for each parameter The training results using RF are presented in Figure 3 for the entire beam and sector 9 , respectively.

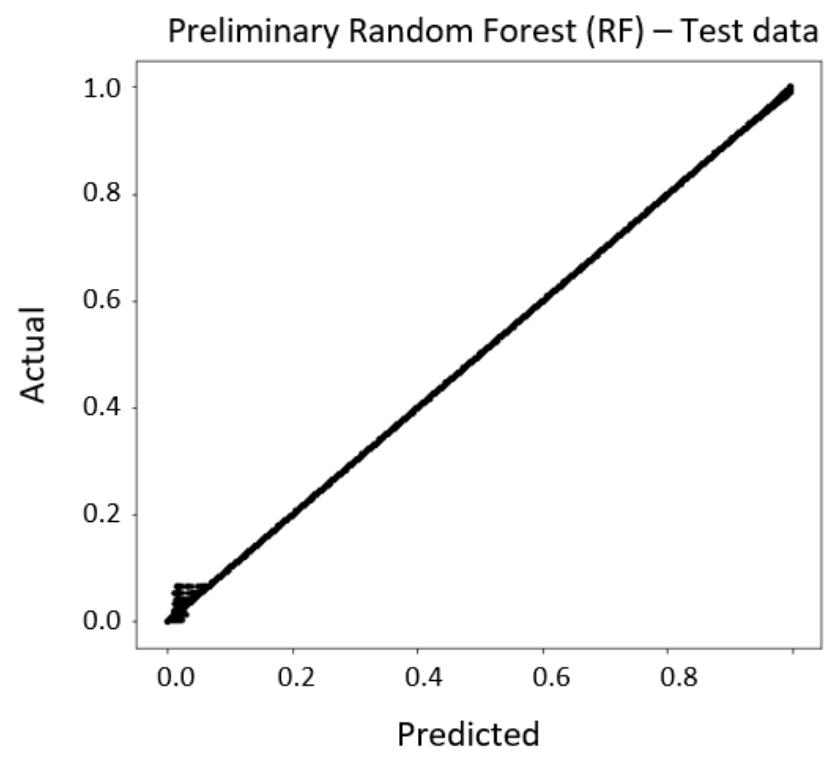

a)

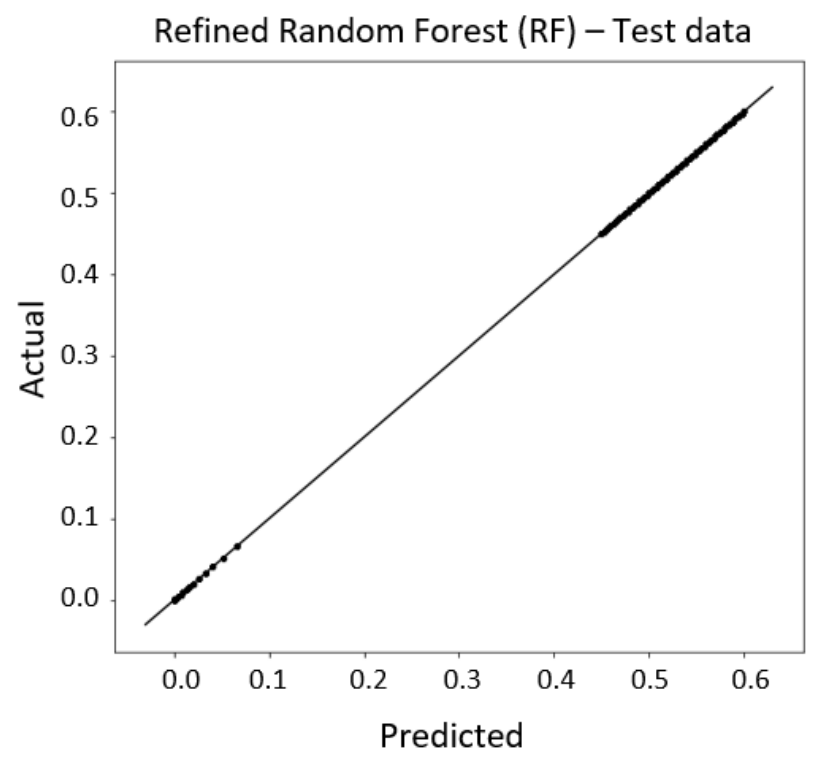

b)

Figure 3. Random forest training results; (a) obtained for coarse estimation involving all input data; (b) obtained for accurate localization involving the Sector 9 input data.

\subsection{Artificial Neural Networks}

Numerous studies have shown the versatility and power of ANNs when applied to different computational tasks such as prediction or classification in many real-world applications [32]. In particular, they are universal approximators capable of detecting nonlinearities in an n-dimensional input. This is achieved by including a large number of nonlinear transformations between the input to an output mapping. A typical neural 
network consists of several connected neurons, organized in layers, as shown in Figure 4. A neuron will generally process information coming from its connections using a nonlinear activation function. A neural network is trained to perform a specific function by adjusting the values of the connections between its neurons.

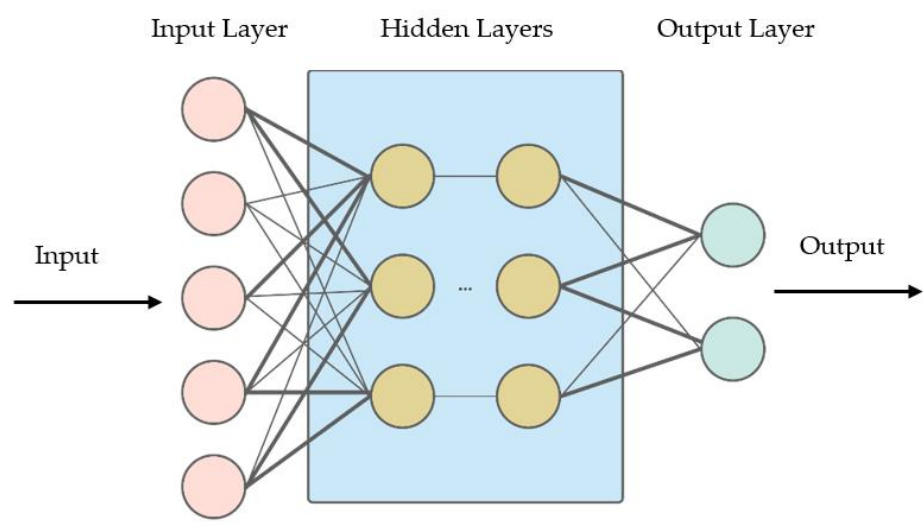

Figure 4. Typical neural network structure.

Feedforward neural networks are ANNs where the topology is organized such that every neuron in one layer projects only onto subsequent layers. This topology excludes thus recurrent connections and essentially means that information flows through the network from one layer to another until it reaches the output.

By using the methodology presented in section 2, we calculated the required data for training. The calculated data involves the INPUT data as the calculated RFS values for the first 8 transverse vibration modes and the TARGET data consisting of 3 values, i.e. crack position, crack severity, and the severity for the weak clamping. If the boundary condition is perfect, the third output is set to zero.

The ANN is developed using Matlab software, as shown in Figure 5. A feedforwardbackpropagation network type is employed, for which we choose: the Bayesian regularization training function, the Levenberg-Marquardt learning function, the Mean squared error (MSE) performance function, and the Hyperbolic tangent sigmoid transfer function.

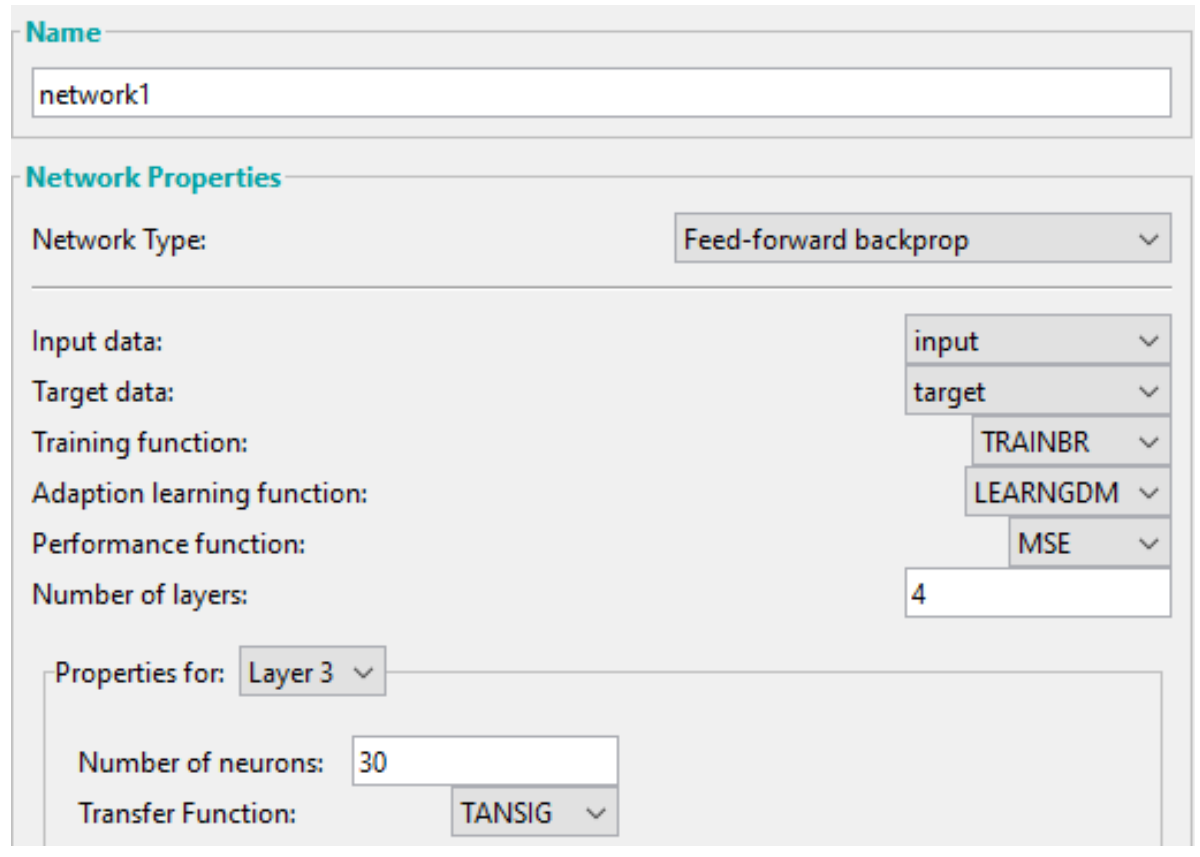

Figure 5. The setup for the training of network 1. 
The ANN, nominated as network 1, consists of 1 input layer with 8 neurons, three hidden layers with 30 neurons each, and an output layer with 3 neurons, see Figure 6.

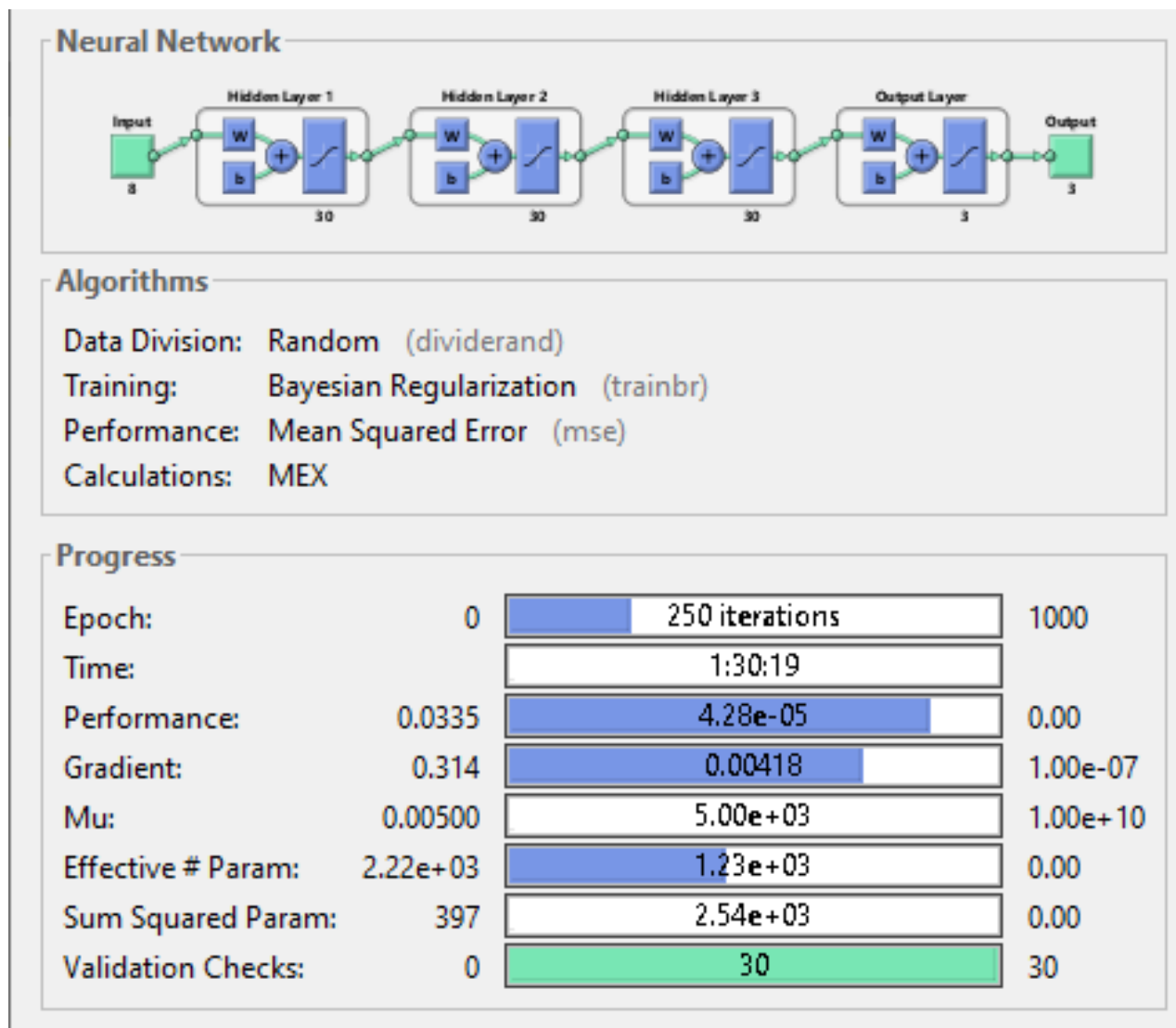

Figure 6. Network 1 configuration and training parameters.

The obtained training performance for network 1 is presented in Figure 7 in comparison with one of the particular networks, section 9 . 

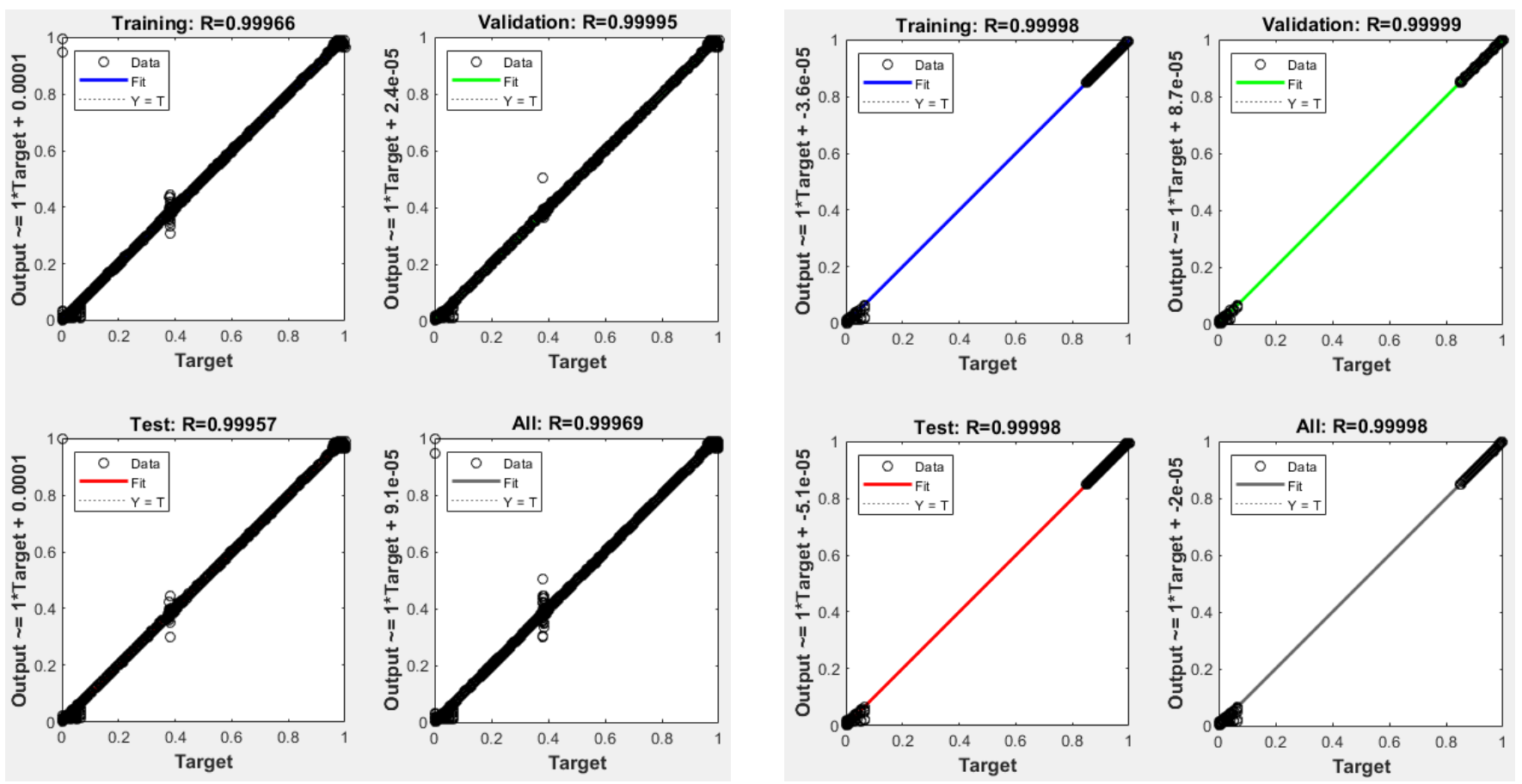

(a)

(b)

Figure 7. Neural network training results: (a) obtained for coarse estimation involving network 1; (b) obtained for accurate localization involving the Sector 9 network.

\subsection{Evaluation of the models}

In this study, two criteria were used for evaluating the accuracy of the models relative to the ones obtained through FEM analysis and experimental tests, $\operatorname{error}(x)$ for the position and error $(\gamma)$ for the severity. The error in the position is

$$
\operatorname{error}(x)=\frac{x(r)-x(e)}{1000} \cdot 100[\%]
$$

Here, $x(r)$ is the real position of the crack expressed in $\mathrm{mm}, x(e)$ is the estimated position using the ML models and 1000 represents the total length of the beam in $\mathrm{mm}$. The severity error is calculated as

$$
\operatorname{error}(\gamma)=\frac{\gamma_{2}(r)-\gamma_{2}(e)}{1} \cdot 100[\%]
$$

In this relation, $\gamma(r)$ is the real severity of the crack, $\gamma(e)$ is the estimated severity of the crack found using the ML models, and 1 represents the maximum value that can be achieved by the crack severity.

In addition to the two above-mentioned errors, we evaluate the capacity of the models to detect weak clamping. The possible responses are false or true.

\section{Numerical validation}

For testing the reliability of the damage detection methods described in the previous section, we first involve the finite element method (FEM). Modal analysis is performed using the ANSYS software. We generate a prismatic steel cantilever beam, like that shown in Figure 8, with dimensions: length $L=1000 \mathrm{~mm}$, width $B=50 \mathrm{~mm}$, and thickness $H=5 \mathrm{~mm}$. The assigned material is S355 JR steel with a modulus of elasticity $E=2 \cdot 10^{5} \mathrm{MPa}$ and a density of $7850 \mathrm{~kg} / \mathrm{m}^{3}$.

The target was to find the first eight natural frequencies for the out-of-plain vibration modes, for the healthy beam and the beam with different damages, respectively. Both ideal clamping and non-ideal clamping are simulated. 


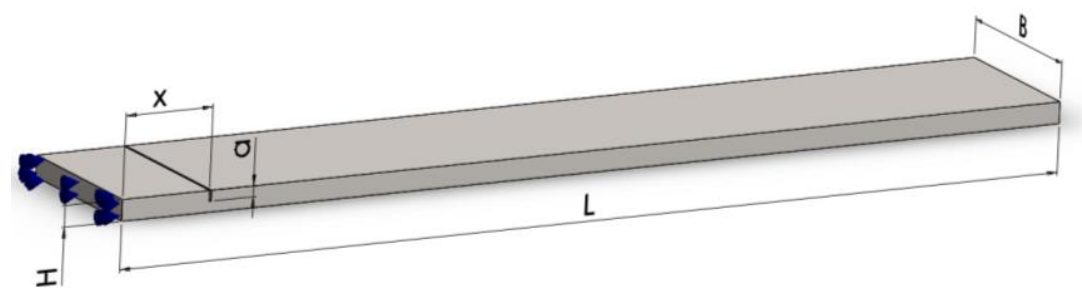

Figure 8. Main dimensions of the considered beam geometry with a transversal crack.

To implement the crack and simulate the effect of the weak clamping, we involve separate elements which are not fixed on one surface, as shown in Figure 9. In this way, we assign the same mass for the beams with damage and non-ideal clamping and yet obtain discontinuities. For simulating real-life scenarios where in most cases measurement data can contain noise, we have also considered different meshing sizes for the damage scenarios to have small differences in the frequency results. The maximum edge size is set to $2 \mathrm{~mm}$ for scenarios 1-5 and $1 \mathrm{~mm}$ for scenarios 6-10.

The FEM model is composed of the beam body and the two parameterized elements used to create discontinuities.

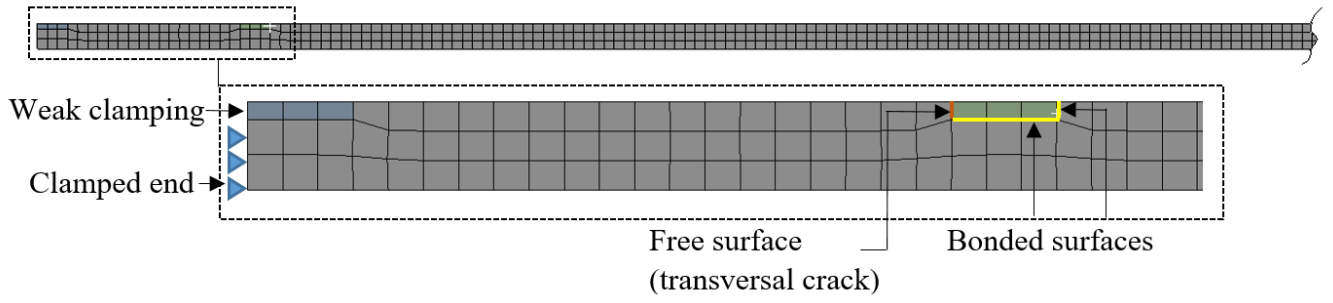

Figure 9. The FEM model of the damaged beam with weak clamping.

The frequencies obtained from the FEM analysis for the undamaged and damaged beam cases are presented in Table 3. The complete dataset is presented in [33]

Table 3. Obtained natural frequencies for the defined damage scenarios.

\begin{tabular}{|c|c|c|c|c|c|c|c|c|c|c|c|}
\hline \multirow{2}{*}{ Scen. } & \multirow{2}{*}{$\begin{array}{c}\text { Crack } \\
\text { pos. } \\
x[\mathrm{~mm}]\end{array}$} & \multirow{2}{*}{$\begin{array}{c}\text { Crack } \\
\text { depth } \\
a_{2}[\mathrm{~mm}]\end{array}$} & \multirow{2}{*}{$\begin{array}{l}\text { Weak } \\
\text { clamp. } \\
\bar{a}_{1}[\%]\end{array}$} & \multicolumn{8}{|c|}{ Natural frequencies obtained for the first 8 modes } \\
\hline & & & & Mode 1 & Mode 2 & Mode 3 & Mode 4 & Mode 5 & Mode 6 & Mode 7 & Mode 8 \\
\hline 0 & & Undama & & 4.09 & 25.627 & 71.757 & 140.63 & 232.53 & 347.46 & 485.47 & 646.59 \\
\hline 1 & 100 & 1 & 0 & 4.081 & 25.606 & 71.745 & 140.631 & 232.472 & 347.229 & 484.947 & 645.751 \\
\hline 2 & 150 & 1 & 0 & 4.082 & 25.620 & 71.754 & 140.552 & 232.257 & 347.024 & 485.083 & 646.448 \\
\hline 3 & 400 & 1 & 0 & 4.087 & 25.601 & 71.708 & 140.588 & 232.186 & 347.447 & 484.904 & 646.092 \\
\hline 4 & 550 & 1 & 0 & 4.089 & 25.588 & 71.739 & 140.478 & 232.383 & 347.238 & 484.950 & 646.435 \\
\hline 5 & 613 & 1 & 0 & 4.089 & 25.593 & 71.686 & 140.609 & 232.189 & 347.401 & 485.079 & 645.855 \\
\hline 6 & 133 & 1 & 20 & 4.073 & 25.568 & 71.624 & 140.323 & 231.864 & 346.328 & 483.945 & 644.903 \\
\hline 7 & 280 & 1.2 & 20 & 4.073 & 25.555 & 71.445 & 140.116 & 231.936 & 346.097 & 483.238 & 644.674 \\
\hline 8 & 410 & 1 & 20 & 4.080 & 25.549 & 71.579 & 140.300 & 231.744 & 346.819 & 483.831 & 645.103 \\
\hline 9 & 570 & 1 & 20 & 4.082 & 25.512 & 71.586 & 140.209 & 231.700 & 346.824 & 483.324 & 645.633 \\
\hline 10 & 962 & 0.6 & 10 & 4.088 & 25.616 & 71.727 & 140.571 & 232.430 & 347.305 & 485.241 & 646.262 \\
\hline
\end{tabular}

The variables used for the studied damage scenarios are the crack location, which is measured from the fixed end, and the proportion in which the clamped end is affected. 
The weak clamping is replicated by adding the separate element of known thickness $\bar{a}_{1}$ to the fixed end. Thus, $\bar{a}_{1}=a_{1} / H$ [\%] replicates the weak clamping. The RFS values obtained for the studied damage scenarios are presented in Table 4, where crack position $x$, crack depth $a_{2}$ and weak clamping $\bar{a}_{1}$ represent the output values.

Table 4. Calculated RFS values for the defined damage scenarios.

\begin{tabular}{|c|c|c|c|c|c|c|c|c|c|c|c|}
\hline \multirow{2}{*}{ Scen. } & \multirow{2}{*}{$\begin{array}{c}\text { Crack } \\
\text { pos. } \\
x[\mathrm{~mm}]\end{array}$} & \multirow{2}{*}{$\begin{array}{c}\text { Crack } \\
\text { depth } \\
a_{2}[\mathrm{~mm}]\end{array}$} & \multirow{2}{*}{$\begin{array}{l}\text { Weak } \\
\text { clamp. } \\
\bar{a}_{1}[\%]\end{array}$} & \multicolumn{8}{|c|}{ RFS for the first 8 modes } \\
\hline & & & & RFS 1 & RFS 2 & RFS 3 & RFS 4 & RFS 5 & RFS 6 & RFS 7 & RFS 8 \\
\hline 1 & 100 & 1 & 0 & 0.002393 & 0.001227 & 0.000540 & 0.000099 & 0.000015 & 0.000075 & 0.000336 & 0.000652 \\
\hline 2 & 150 & 1 & 0 & 0.001899 & 0.000254 & 0.000038 & 0.000552 & 0.001173 & 0.001254 & 0.000798 & 0.000219 \\
\hline 3 & 400 & 1 & 0 & 0.000644 & 0.001020 & 0.000683 & 0.000299 & 0.001480 & 0.000037 & 0.001166 & 0.000771 \\
\hline 4 & 550 & 1 & 0 & 0.000240 & 0.001535 & 0.000256 & 0.001080 & 0.000632 & 0.000640 & 0.001072 & 0.000240 \\
\hline 5 & 613 & 1 & 0 & 0.000141 & 0.001343 & 0.000991 & 0.000146 & 0.001467 & 0.000171 & 0.000805 & 0.001136 \\
\hline 6 & 133 & 1 & 20 & 0.002224 & 0.000466 & 0.000003 & 0.000336 & 0.001015 & 0.001417 & 0.00131 & 0.00077 \\
\hline 7 & 280 & 1.2 & 20 & 0.004237 & 0.002804 & 0.004348 & 0.003654 & 0.002554 & 0.003922 & 0.004598 & 0.002963 \\
\hline 8 & 410 & 1 & 20 & 0.002522 & 0.003063 & 0.002483 & 0.002346 & 0.003381 & 0.001844 & 0.003377 & 0.002300 \\
\hline 9 & 570 & 1 & 20 & 0.000218 & 0.00167 & 0.000507 & 0.000837 & 0.00116 & 0.000208 & 0.001627 & 0.00002 \\
\hline 10 & 962 & 0.6 & 10 & 0.000412 & 0.000412 & 0.000414 & 0.000419 & 0.000429 & 0.000446 & 0.000472 & 0.000507 \\
\hline
\end{tabular}

The outputs obtained using the two ML methods based on the RFS values (Table 4) are presented in Tables 5-8. Table 5 contains the results from the preliminary ML random forest model, and Table 6 the results obtained using the refined random forest model. In Table 7 we present the results obtained involving coarse estimation with network 1 , and in Table 8 are the results obtained with the specific ANN networks chosen for the particular cases after the coarse estimation. The severity of the cracks with depth $a=1 \mathrm{~mm}, a=1.2 \mathrm{~mm}$, and $a=0.6 \mathrm{~mm}$ is: $\gamma_{2}(1)=0.0033459, \gamma_{2}(1.2)=0.0051239$ and $\gamma_{2}(0.6)=0.0011911$.

Table 5. Results obtained using Random forest rough model - first step.

\begin{tabular}{|c|c|c|c|c|c|c|c|c|}
\hline & FEM Scen & harios & & Rand & lom Fores & t prelimi & nary output & \\
\hline Scen. & Position & Severity & Position & Severity & $\begin{array}{c}\text { Position } \\
\text { error }\end{array}$ & $\begin{array}{c}\text { Severity } \\
\text { Error }\end{array}$ & Weak cla & ping \\
\hline 1 & 100 & 0.0033459 & 99.5 & 0.0028 & 0.05 & 0.05 & Not detected & True \\
\hline 2 & 150 & 0.0033459 & 151.6 & 0.0029 & 0.16 & 0.04 & Not detected & True \\
\hline 3 & 400 & 0.0033459 & 458.0 & 0.0024 & 5.80 & 0.09 & Not detected & True \\
\hline 4 & 550 & 0.0033459 & 515.8 & 0.0028 & 3.42 & 0.05 & Not detected & True \\
\hline 5 & 613 & 0.0033459 & 602.5 & 0.0028 & 1.05 & 0.05 & Not detected & True \\
\hline 6 & 133 & 0.0033459 & 138.1 & 0.0027 & 0.51 & 0.06 & Not detected & False \\
\hline 7 & 280 & 0.0051239 & 204.9 & 0.0068 & 7.51 & 0.17 & Detected & True \\
\hline 8 & 410 & 0.0033459 & 365.1 & 0.0044 & 4.49 & 0.11 & Detected & True \\
\hline 9 & 570 & 0.0033459 & 547.3 & 0.0063 & 2.27 & 0.30 & Detected & True \\
\hline 10 & 962 & 0.0011911 & 941.1 & 0.0129 & 2.09 & 1.17 & Not detected & False \\
\hline
\end{tabular}

One can observe that, after the first check, the largest error in estimating the crack position is $7.51 \%$, which is unacceptable. The severity is precisely found, the errors being 
less than $1.17 \%$. The method was not able to indicate correctly if the clamping is non-ideal. In consequence, this machine learning method is not qualified to assess the crack position and severity at this stage.

Table 6. Results obtained using Random forest enhanced model - second step.

\begin{tabular}{|c|c|c|c|c|c|c|c|c|}
\hline \multicolumn{3}{|c|}{ FEM Scenarios } & \multicolumn{6}{|c|}{ Refined Random Forest output } \\
\hline Scen. & $\begin{array}{c}\text { Position } \\
\text { [mm] }\end{array}$ & $\begin{array}{c}\text { Severity } \\
\gamma_{2}\left(\mathbf{a}_{2}\right)\end{array}$ & $\begin{array}{c}\text { Position } \\
\text { [mm] }\end{array}$ & $\begin{array}{c}\text { Severity } \\
\gamma_{2}\left(\mathbf{a}_{2}\right)\end{array}$ & $\begin{array}{c}\text { Position } \\
\text { error } \\
{[\%]}\end{array}$ & $\begin{array}{c}\text { Severity } \\
\text { Error } \\
{[\%]}\end{array}$ & Weak clar & ping \\
\hline 1 & 100 & 0.0033459 & 97.7 & 0.0030 & 0.23 & 0.03 & Detected & False \\
\hline 2 & 150 & 0.0033459 & 148.3 & 0.0029 & 0.17 & 0.04 & Detected & False \\
\hline 3 & 400 & 0.0033459 & 400.0 & 0.0025 & 0 & 0.08 & Not detected & True \\
\hline 4 & 550 & 0.0033459 & 539.6 & 0.0028 & 1.04 & 0.05 & Detected & False \\
\hline 5 & 613 & 0.0033459 & 615.3 & 0.0026 & 0.23 & 0.07 & Not detected & True \\
\hline 6 & 133 & 0.0033459 & 136.3 & 0.0024 & 0.33 & 0.09 & Not detected & False \\
\hline 7 & 280 & 0.0051239 & 303.4 & 0.0087 & 2.34 & 0.36 & Detected & True \\
\hline 8 & 410 & 0.0033459 & 410.1 & 0.0046 & 0.01 & 0.13 & Detected & True \\
\hline 9 & 570 & 0.0033459 & 570.1 & 0.0058 & 0.01 & 0.25 & Detected & True \\
\hline 10 & 962 & 0.0011911 & 976.3 & 0.010 & 1.43 & 0.88 & Detected & True \\
\hline
\end{tabular}

Localization after the second check with the RF method is accurate, errors being below $1 \%$. Estimating the severity of the defect is done also with small errors, up to $0.88 \%$. As shown in Table 6, weak clamping is detected incorrectly for some damage scenarios.

Assessing the crack with the ANN method is also made in two steps. First, a coarse estimation is made with network 1, which considers the locations along the entire beam. Here, we coarsely estimate the damage location and choose the segment on which the crack is supposed to exist and perform a second check for the network trained for scenarios available on this segment.

Table 7. Results obtained using network 1 - coarse estimation.

\begin{tabular}{|c|c|c|c|c|c|c|c|c|}
\hline & FEM Scen & larios & & Coarse & network & INetwork & 1 output & \\
\hline Scen. & Position & Severity & Position & Severity & $\begin{array}{c}\text { Position } \\
\text { error }\end{array}$ & $\begin{array}{c}\text { Severity } \\
\text { Error }\end{array}$ & Weak clam & oing \\
\hline 1 & 100 & 0.0033459 & 99.6 & 0.0027 & 0.04 & 0.06 & Not detected & False \\
\hline 2 & 150 & 0.0033459 & 147.7 & 0.0023 & 0.23 & 0.10 & Not detected & False \\
\hline 3 & 400 & 0.0033459 & 392.9 & 0.0011 & 0.71 & 0.22 & Not detected & False \\
\hline 4 & 550 & 0.0033459 & 553.9 & 0.0031 & 0.39 & 0.02 & Not detected & False \\
\hline 5 & 613 & 0.0033459 & 604.4 & 0.0022 & 0.86 & 0.11 & Not detected & False \\
\hline 6 & 133 & 0.0033459 & 132.9 & 0.0047 & 0.01 & 0.14 & Detected & True \\
\hline 7 & 280 & 0.0051239 & 261.5 & 0.0098 & 1.85 & 0.47 & Detected & True \\
\hline 8 & 410 & 0.0033459 & 377.5 & 0.0033 & 3.25 & 0.00 & Detected & True \\
\hline 9 & 570 & 0.0033459 & 569.1 & 0.0072 & 0.09 & 0.39 & Detected & True \\
\hline 10 & 962 & 0.0011911 & 971 & 0.0134 & 0.9 & 1.22 & Detected & True \\
\hline
\end{tabular}

After the first check, the largest error obtained is $3.3 \%$ for the position, and small errors were obtained for the crack severity. The model wrongly detects non-ideal clamping for the first five damage scenarios. But, in this stage, we can estimate the segment on which the crack occurred with high confidence. 
Table 8. Results obtained using the particular networks - fine estimation.

\begin{tabular}{|c|c|c|c|c|c|c|c|c|c|}
\hline \multicolumn{3}{|c|}{ FEM Scenarios } & \multicolumn{7}{|c|}{ Accuracy enhanced network output } \\
\hline Scen. & Position & Severity & Network & Position & Severity & $\begin{array}{c}\text { Position } \\
\text { error }\end{array}$ & $\begin{array}{c}\text { Severity } \\
\text { Error }\end{array}$ & Weak clam & ing \\
\hline 1 & 100 & 0.0033459 & Sector 1 & 99.6 & 0.003332 & 0.04 & 0.00 & Not detected & True \\
\hline 2 & 150 & 0.0033459 & Sector 2 & 148.9 & 0.003174 & 0.11 & 0.02 & Not detected & True \\
\hline 3 & 400 & 0.0033459 & Sector 4 & 398.3 & 0.003031 & 0.17 & 0.03 & Not detected & True \\
\hline 4 & 550 & 0.0033459 & Sector 5 & 554.7 & 0.002947 & 0.47 & 0.04 & Not detected & False \\
\hline 5 & 613 & 0.0033459 & Sector 6 & 612.6 & 0.002936 & 0.04 & 0.04 & Not detected & True \\
\hline 6 & 133 & 0.0033459 & Sector 1 & 132.3 & 0.003385 & 0.07 & 0.00 & Detected & True \\
\hline 7 & 280 & 0.0051239 & Sector 3 & 280.8 & 0.004469 & 0.08 & 0.07 & Detected & True \\
\hline 8 & 410 & 0.0033459 & Sector 4 & 409.4 & 0.003086 & 0.06 & 0.03 & Detected & True \\
\hline 9 & 570 & 0.0033459 & Sector 6 & 570.4 & 0.003353 & 0.04 & 0.00 & Detected & True \\
\hline 10 & 962 & 0.0011911 & Sector 9 & 962.2 & 0.001419 & 0.02 & 0.02 & Detected & True \\
\hline
\end{tabular}

Knowing the approximate position of the crack we can select the appropriate segment and use the network trained for this particular segment. The results obtained in the second step are given in Table 8. One can observe that now the largest error obtained is $0.5 \%$ for the position and $0.07 \%$ for the crack severity. In addition, the model can detect non-ideal clamping with high accuracy. These results allow us to conclude that the twostep ANN method is more efficient in crack assessment.

\section{Experimental validation}

To validate the developed ANN models, experimental studies were carried out by measuring the first natural frequencies for five steel beams with the dimensions indicated in the previous section. In the first 5 damage scenarios, the beams were perfectly clamped. Furthermore, the capability of the developed method to detect non-ideal clamping was also tested for the damaged beams by mounting rubber blocks between the jaws of the vise and the beam (Figure 10).

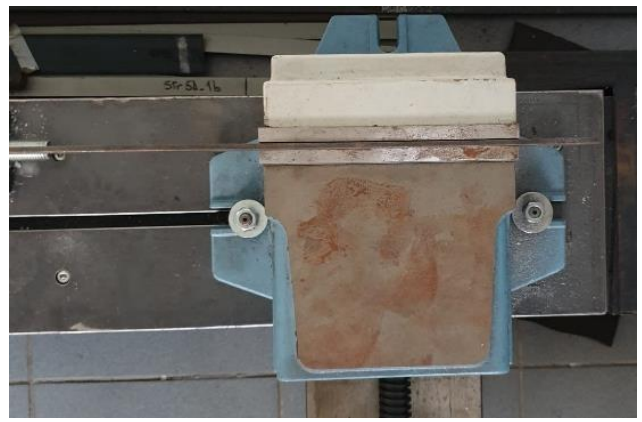

a)

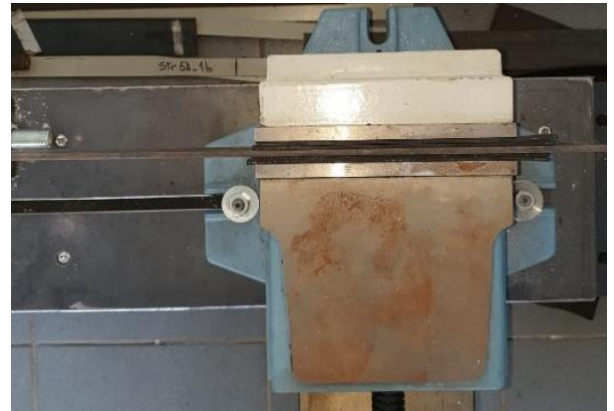

b)

Figure 10. Clamping system: (a) rigid clamping obtained by direct fixing in the vise; (b) weak clamping obtained by intercalating rubber layers.

The acceleration signals are recorded with a Kistler 8772 accelerometer mounted on the tested beam. It transmits the measured signal through the analog-to-digital conversion module NI9234 and a compact chassis NIcDAQ-9175 to a connected laptop. To excite the beam at the desired resonant frequencies, see the procedure in [34], an audio excitation 
system consisting of a loudspeaker, amplifier, and dedicated software installed on a second laptop is used. The experimental setup is shown in Figure 11.

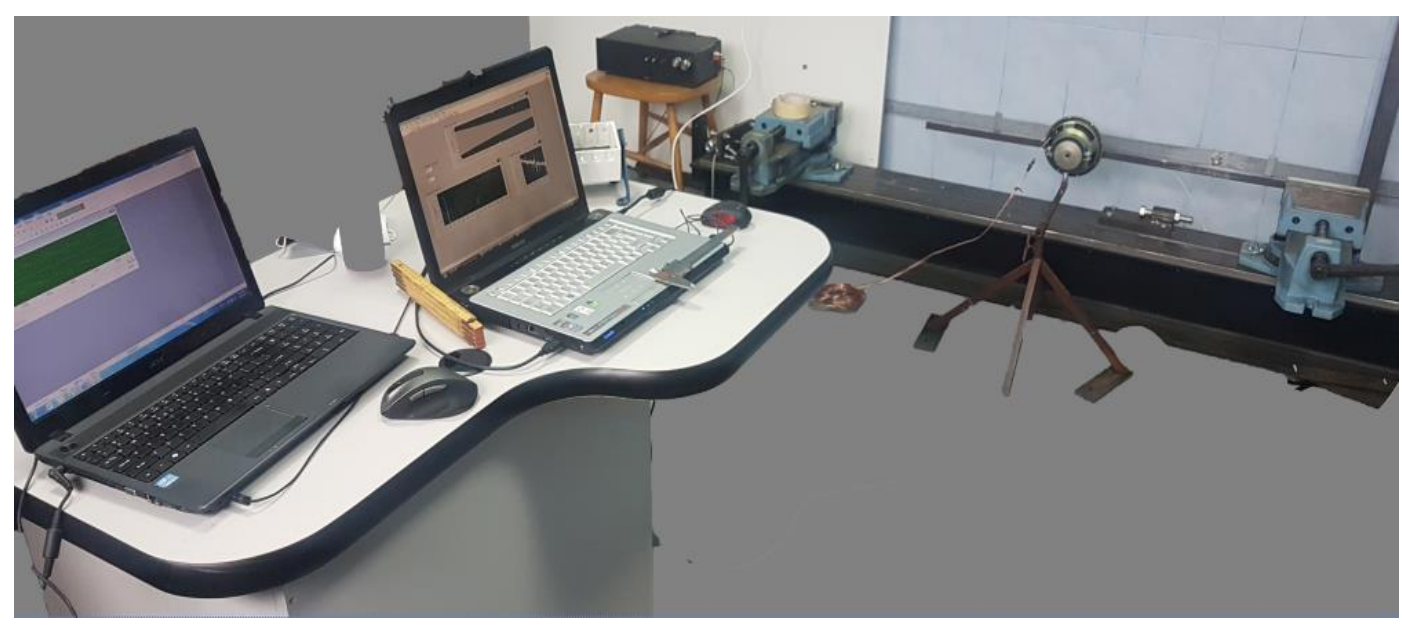

Figure 11. Experimental setup.

The natural frequencies are estimated one by one, with high accuracy, after a procedure described in [35]. The Python code to estimate the frequencies is given in [36].

\subsection{Perfect clamping experiments}

In the first experimental study, each beam is mounted into a vise, thus achieving a rigid clamping. The resulted frequencies for the undamaged beams are shown in Table 9.

Table 9. Frequencies estimated for the undamaged beams.

\begin{tabular}{ccccccccc}
\hline \multirow{2}{*}{ Test beam } & Mode 1 & Mode 2 & Mode 3 & Mode 4 & Mode 5 & Mode 6 & Mode 7 & Mode 8 \\
\hline Beam 1 & 4,035 & 25,284 & 70,970 & 139,090 & 230,336 & 344,196 & 481,809 \\
Beam 2 & 4,060 & 25,439 & 71,426 & 139,902 & 231,038 & 344,750 & 482,503 \\
Beam 3 & 4,034 & 25,341 & 71,064 & 139,15 & 230,138 & 341,868 & 480,773 & 641,823 \\
Beam 4 & 4,030 & 25,367 & 71,213 & 139,342 & 230,295 & 343,254 & 480,795 & 639,510 \\
Beam 5 & 4,044 & 25,482 & 71,287 & 139,42 & 228,528 & 344,177 & 481,213 & 641,114 \\
\hline
\end{tabular}

Several damage scenarios are generated by saw cutting each of the five beams at different positions and depths thus replicating a transversal crack. The position and depth for every scenario are according to Table 10.

Table 10. Estimated natural frequencies for the test beams containing a crack of known position and depth.

\begin{tabular}{|c|c|c|c|c|c|c|c|c|c|c|}
\hline \multirow{2}{*}{$\begin{array}{c}\text { Test } \\
\text { beam }\end{array}$} & \multirow{2}{*}{$\begin{array}{c}\text { Crack } \\
\text { position }\end{array}$} & \multirow{2}{*}{$\begin{array}{l}\text { Crack } \\
\text { depth }\end{array}$} & \multicolumn{8}{|c|}{ Natural frequencies $[\mathrm{Hz}]$} \\
\hline & & & Mode 1 & Mode 2 & Mode 3 & Mode 4 & Mode 5 & Mode 6 & Mode 7 & Mode 8 \\
\hline 1 & 98 & 2,5 & 3.952 & 25.086 & 70.854 & 139.086 & 229.859 & 342.143 & 477.105 & 633.647 \\
\hline 2 & 310 & 1,25 & 4.053 & 25.422 & 71.259 & 139.818 & 230.913 & 343.835 & 481.913 & 641.729 \\
\hline 3 & 569 & 2,5 & 4.024 & 24.904 & 70.707 & 137.883 & 227.494 & 340.994 & 472.791 & 636.758 \\
\hline 4 & 126 & 2,5 & 4.200 & 25.226 & 71.208 & 138.982 & 228.245 & 338.441 & 473.771 & 632.801 \\
\hline 5 & 759 & 2,5 & 4.043 & 25.343 & 70.051 & 137.012 & 227.517 & 343.834 & 475.326 & 630.614 \\
\hline
\end{tabular}

By using the measured frequencies for the beams in an undamaged and damaged state, we obtain the RFS values with Eq.2; the results are presented in Table 11. 
Table 11. Calculated RFS value for each test beam.

\begin{tabular}{ccccccccccc}
\hline $\begin{array}{c}\text { Test } \\
\text { beam }\end{array}$ & $\begin{array}{c}\text { Crack } \\
\text { position }\end{array}$ & $\begin{array}{c}\text { Crack } \\
\text { depth }\end{array}$ & Mode 1 & Mode 2 & Mode 3 & Mode 4 & Mode 5 & Mode 6 & Mode 7 & Mode 8 \\
\hline 1 & 98 & 2,5 & 0.020610 & 0.007828 & 0.001629 & 0.000031 & 0.002070 & 0.005964 & 0.009762 & 0.011873 \\
2 & 310 & 1,25 & 0.001795 & 0.000660 & 0.002334 & 0.000600 & 0.000542 & 0.002654 & 0.001223 & 0.000146 \\
3 & 569 & 2,5 & 0.002382 & 0.017252 & 0.005019 & 0.009109 & 0.011488 & 0.002556 & 0.016603 & 0.000017 \\
4 & 126 & 2,5 & 0.023458 & 0.005550 & 0.000064 & 0.002581 & 0.008901 & 0.014021 & 0.014610 & 0.010491 \\
5 & 759 & 2,5 & 0.000288 & 0.005461 & 0.017336 & 0.017272 & 0.004422 & 0.000996 & 0.012234 & 0.016377 \\
\hline
\end{tabular}

The RFS data is fed to the coarse ML models to obtain the estimated crack position and severity for the five damage scenarios. The results obtained after the first estimation are shown in Tables 12 for the RF model and in Table 13 for the ANN model.

Table 12. Results obtained using the preliminary Random Forest model

\begin{tabular}{ccc|cccccc}
\hline \multicolumn{6}{c|}{ Damage Scenarios } & \multicolumn{5}{c}{ Preliminary Random Forest } \\
\hline Scen. & $\begin{array}{c}\text { Position } \\
{[\mathbf{m m}]}\end{array}$ & $\begin{array}{c}\text { Severity } \\
\gamma_{2}(\mathbf{a})\end{array}$ & $\begin{array}{c}\text { Position } \\
{[\mathbf{m m}]}\end{array}$ & $\begin{array}{c}\text { Severity } \\
\gamma_{2}(\mathbf{a})\end{array}$ & $\begin{array}{c}\text { Position } \\
\text { error } \\
{[\%]}\end{array}$ & $\begin{array}{c}\text { Severity } \\
\text { Error } \\
{[\%]}\end{array}$ & Weak clamping \\
\hline 1 & 98 & 0.026224 & 96.99 & 0.0264 & 0.10 & 0.02 & Not detected & True \\
2 & 310 & 0.005124 & 309.85 & 0.0051 & 0.01 & 0.00 & Not detected & True \\
3 & 569 & 0.026224 & 564.87 & 0.0331 & 0.41 & 0.69 & Not detected & True \\
4 & 126 & 0.026224 & 125.42 & 0.0329 & 0.06 & 0.67 & Not detected & True \\
5 & 759 & 0.026224 & 758.25 & 0.0329 & 0.08 & 0.67 & Not detected & True \\
\hline
\end{tabular}

Table 13. Results obtained using the ANN - Network 1

\begin{tabular}{ccc|cccccc}
\hline \multicolumn{6}{c|}{ Damage Scenarios } & \multicolumn{5}{c}{ Coarse ANN - Network 1 output } \\
\hline Scen. & $\begin{array}{c}\text { Position } \\
{[\mathbf{m m}]}\end{array}$ & $\begin{array}{c}\text { Severity } \\
\gamma_{2}(\mathbf{a})\end{array}$ & $\begin{array}{c}\text { Position } \\
{[\mathbf{m m}]}\end{array}$ & $\begin{array}{c}\text { Severity } \\
\gamma_{2}(\mathbf{a})\end{array}$ & $\begin{array}{c}\text { Position } \\
\text { error } \\
{[\%]}\end{array}$ & $\begin{array}{c}\text { Severity } \\
\text { Error } \\
{[\%]}\end{array}$ & Weak clamping \\
\hline 1 & 98 & 0.026224 & 97.8 & 0.0282 & 0.02 & 0.20 & Not detected & False \\
2 & 310 & 0.005124 & 313.2 & 0.0042 & 0.32 & 0.09 & Not detected & False \\
3 & 569 & 0.026224 & 567.2 & 0.0323 & 0.18 & 0.61 & Not detected & False \\
4 & 126 & 0.026224 & 126 & 0.0337 & 0.00 & 0.75 & Not detected & False \\
5 & 759 & 0.026224 & 757.8 & 0.0331 & 0.12 & 0.69 & Not detected & False \\
\hline
\end{tabular}

We identify the segments in which cracks were found and select the corresponding networks. Afterward, by employing the enhanced ML models we obtain more precise results, as presented in Table 14 for the refined Random forest model and in Table 15 for the enhanced ANN model. 
Table 14. Obtained results using the refined Random Forest model

\begin{tabular}{ccc|cccccc}
\hline \multicolumn{3}{c|}{ Damage Scenarios } & \multicolumn{5}{c}{ Refined Random Forest } \\
\hline Scen. & $\begin{array}{c}\text { Position } \\
{[\mathbf{m m}]}\end{array}$ & $\begin{array}{c}\text { Severity } \\
\gamma_{\mathbf{2}}(\mathbf{a} \text { 2) }\end{array}$ & $\begin{array}{c}\text { Position } \\
{[\mathbf{m m}]}\end{array}$ & $\begin{array}{c}\text { Severity } \\
\gamma_{\mathbf{2}}(\mathbf{a} \text { ) })\end{array}$ & $\begin{array}{c}\text { Position } \\
\text { error } \\
{[\%]}\end{array}$ & $\begin{array}{c}\text { Severity } \\
\text { Error } \\
{[\%]}\end{array}$ & Weak clamping \\
\hline 1 & 98 & 0.026224 & 99.40 & 0.0260 & 0.14 & 0.02 & Not detected & True \\
2 & 310 & 0.005124 & 309.73 & 0.0049 & 0.03 & 0.02 & Not detected & True \\
3 & 569 & 0.026224 & 568.22 & 0.0327 & 0.08 & 0.65 & Not detected & True \\
4 & 126 & 0.026224 & 127.84 & 0.0329 & 0.18 & 0.67 & Not detected & True \\
5 & 759 & 0.026224 & 758.19 & 0.0328 & 0.08 & 0.66 & Not detected & True \\
\hline
\end{tabular}

Table 15. Obtained results using the precision sector ANN

\begin{tabular}{|c|c|c|c|c|c|c|c|}
\hline \multicolumn{3}{|c|}{ Damage Scenarios } & \multicolumn{5}{|c|}{ Enhanced network output } \\
\hline Scen. & $\begin{array}{c}\text { Position } \\
\text { [mm] }\end{array}$ & $\begin{array}{c}\text { Severity } \\
\gamma_{2}\left(\mathrm{a}_{2}\right)\end{array}$ & $\begin{array}{c}\text { Position } \\
\text { [mm] }\end{array}$ & $\begin{array}{c}\text { Severity } \\
\gamma_{2}\left(\mathrm{a}_{2}\right)\end{array}$ & $\begin{array}{c}\text { Position } \\
\text { error } \\
{[\%]}\end{array}$ & $\begin{array}{c}\text { Severity } \\
\text { Error } \\
{[\%]}\end{array}$ & Weak clamping \\
\hline 1 & 98 & 0.026224 & 98 & 0.0275 & 0.00 & 0.13 & Not detected \\
\hline 2 & 310 & 0.005124 & 310 & 0.0054 & 0.00 & 0.03 & Not detected True \\
\hline 3 & 569 & 0.026224 & 568 & 0.0343 & 0.10 & 0.81 & Not detected True \\
\hline 4 & 126 & 0.026224 & 126 & 0.0343 & 0.00 & 0.81 & Not detected True \\
\hline 5 & 759 & 0.026224 & 758 & 0.0343 & 0.10 & 0.81 & Not detected True \\
\hline
\end{tabular}

One can observe from Tables 14 and 15 that, for the case of ideal clamping, the damage location and severity are found with high accuracy irrespective of the employed method. Remarkably, ideal clamping is detected in all cases.

\subsection{Improper clamping experiments}

This study concerns the ability of the proposed approach to detect cracks in two different cases:

- The structure has initially ideal boundary conditions, but after a while, a crack occurs and an alteration of the fixing system is present, i.e. the clamping becomes non-ideal

- The structure has from the beginning non-ideal boundary conditions, so the fixing system remains unchanged, and is affected after a while by a crack.

The experiment is made for test beam 1. Improper clamping was ensured by mounting rubber blocks between the jaws of the vise and the test beam, as shown in Figure $10 \mathrm{~b}$. The crack is generated at distance $x_{2}=98 \mathrm{~mm}$ and has depth $a_{2}=2.5 \mathrm{~mm}$, The natural frequencies were obtained from the measured data. The results for the undamaged beam with ideal and non-ideal clamping, as well as the frequencies for the test beam 1 with non-ideal fixing and a crack are shown in Table 16. 
Table 16. Estimated natural frequencies.

\begin{tabular}{lccccccccc}
\hline Test & Crack & Crack & & \multicolumn{7}{c}{ Calculated natural frequencies [Hz] } \\
beam position & depth & Mode 1 & Mode 2 & Mode 3 & Mode 4 & Mode 5 & Mode 6 & Mode 7 & Mode 8 \\
\hline $\begin{array}{l}\text { Undamaged with ideal } \\
\text { clamping }\end{array}$ & 4,035 & 25,284 & 70,970 & 139,090 & 230,336 & 344,196 & 481,809 & 641,261 \\
Undamaged with non- & 4.0051 & 25.111 & 70.48 & 138.95 & 229.21 & 341.18 & 476.93 & 635.49 \\
$\begin{array}{l}\text { ideal clamping } \\
\quad 1 \quad 98\end{array} \quad 2,5$ & 3.926 & 24.935 & 70.420 & 138.211 & 228.362 & 339.873 & 474.057 & 630.080 \\
\hline
\end{tabular}

The calculated RFS values are presented in Table 17, first for the undamaged beam with ideal fixing (case 1i) and afterward, for the undamaged beam with initially non-ideal fixing (case $1 n-i)$, In both cases, the damaged beam has non-ideal clamping.

Table 17. Calculated RFS values for the improperly clamped damaged beam.

\begin{tabular}{cccccccccccc}
\hline $\begin{array}{c}\text { Test } \\
\text { beam }\end{array}$ & $\begin{array}{c}\text { Crack } \\
\text { position }\end{array}$ & $\begin{array}{c}\text { Crack } \\
\text { depth }\end{array}$ & Mode 1 & Mode 2 & Mode 3 & Mode 4 & Mode 5 & Mode 6 & Mode 7 & Mode 8 \\
\hline $1 \mathrm{i}$ & 98 & 2,5 & 0.026955 & 0.013803 & 0.007749 & 0.006322 & 0.008572 & 0.012559 & 0.016089 & 0.017436 \\
\hline $1 \mathrm{n}-\mathrm{i}$ & 98 & 2,5 & 0.019749 & 0.007008 & 0.000851 & 0.005318 & 0.003699 & 0.003830 & 0.006023 & 0.008513 \\
\hline
\end{tabular}

The crack assessment is made employing the two ML methods, in compliance with the two evaluation stages. The results for case $1 i$ are presented in Table 18, while the results for case $1 n-i$ are presented in table 19 .

Table 18. Crack assessment for a damaged beam with non-ideal clamping for the case the undamaged beam had initially an ideal fixing (case 1i).

\begin{tabular}{ccc|cccccc}
\hline \multicolumn{3}{c|}{ Damage Scenario } & \multicolumn{5}{c}{ Results obtained with the ML models } \\
\hline Scen. & $\begin{array}{c}\text { Position } \\
{[\mathbf{m m}]}\end{array}$ & $\begin{array}{c}\text { Severity } \\
\gamma_{\mathbf{2}}(\mathbf{a})\end{array}$ & $\begin{array}{c}\text { Position } \\
{[\mathbf{m m}]}\end{array}$ & $\begin{array}{c}\text { Severity } \\
\gamma_{\mathbf{2}}\left(\mathbf{a}_{2}\right)\end{array}$ & $\begin{array}{c}\text { Position } \\
\text { error } \\
{[\%]}\end{array}$ & $\begin{array}{c}\text { Severity } \\
\text { Error } \\
{[\%]}\end{array}$ & Weak clamping \\
\hline First-step RF & & & 129.36 & 0.038300 & 3.14 & 1.21 & Detected & True \\
Second-step RF & \multirow{2}{*}{98} & 0.026224 & 92.68 & 0.039000 & 0.53 & 1.28 & Detected & True \\
First-step ANN & & & 102.07 & 0.031283 & 0.41 & 0.51 & Detected & True \\
Second-step ANN & & & 99.13 & 0.027038 & 0.11 & 0.08 & Detected & True \\
\hline
\end{tabular}

Table 19. Crack assessment for a damaged beam with non-ideal (but unchanged) clamping (case 1ii).

\begin{tabular}{ccc|cccccc}
\hline \multicolumn{3}{c|}{ Damage Scenarios } & \multicolumn{5}{c}{ Results obtained with the ML models } \\
\hline Scen. & $\begin{array}{c}\text { Position } \\
{[\mathrm{mm}]}\end{array}$ & $\begin{array}{c}\text { Severity } \\
\gamma_{2}(\mathbf{a})\end{array}$ & $\begin{array}{c}\text { Position } \\
{[\mathbf{m m}]}\end{array}$ & $\begin{array}{c}\text { Severity } \\
\gamma_{2}\left(\mathbf{a}_{2}\right)\end{array}$ & $\begin{array}{c}\text { Position } \\
\text { error } \\
{[\%]}\end{array}$ & $\begin{array}{c}\text { Severity } \\
\text { Error } \\
{[\%]}\end{array}$ & Weak clamping \\
\hline First-step RF & & & 82.54 & 0.0353 & 1.546 & -0.9076 & Not detected & True \\
Second-step RF & \multirow{2}{*}{98} & 0.026224 & 32.05 & 0.0292 & 6.595 & -0.2976 & Not detected & True \\
First-step ANN & & & 74.63 & 0.0247 & 2.337 & 0.1524 & Not detected & False \\
Second-step ANN & & & 96.4 & 0.0247 & 0.16 & 0.1524 & Not detected & True \\
\hline
\end{tabular}

Reviewing Tables 18 and 19, we can conclude that for the beam with changing boundary conditions the crack position and severity were found accurately after the second step. The best result is obtained using the ANN, which located the crack with an error of $0.11 \%$ and found the severity with an error below $0.1 \%$.

The changed boundary conditions are detected with both ML methods. Dissimilar, the case when the beam had initially a weak fixing was properly solved just by the ANN 
method. The errors accuracy, in this case, is sustained by errors less than $0.16 \%$, for position and severity as well. Both ML methods did not find an alteration of the fixing in the vise, thus we consider the response Not detected as true.

\title{
6. Conclusions
}

In this paper, we apply RF and ANN to identify the crack location and severity in a steel cantilever beam with rectangular cross-section. The beam has different levels of fixing, including ideal and non-ideal clamping, and we also aim to find if the fixing condition changes. To train the network we use as inputs the RFS found applying an original method and as the crack parameters and the fixing condition, respectively. To improve the accuracy of the assessment method, we perform crack assessment in two steps: (i) in the first step we apply coarse estimation, for the network trained for all damage scenarios - here the region in which the crack occurred is identified; (ii) in the second step we apply a fine estimation, for the network trained for a specific segment of the beam which includes the damaged region - here the crack position and severity are found, along with the changes in clamping, if any.

Both ML methods were able to learn and classify new data with characteristics comparable with that of the training data. After training, the networks are successfully used to assess other scenarios, which come from FEM simulation and laboratory experiments. We found that, for all cases, the ability to accurately detect the crack location and severity increases when using assessment in two steps. After the second step, the errors in locating the crack are below $\mathrm{xx} \%$, and the errors in assessing the crack severity are less than $\mathrm{yy} \%$. The ANN leads to better results. The smallest crack we found had $0.6 \mathrm{~mm}$ depth, in a beam with a thickness of $5 \mathrm{~mm}$. We did not search after smaller crack depths because of the training data we produced and knowing that the ANN method is more efficient in interpolation than in extrapolation.

Further investigation should be conducted to evaluate the ability of the proposed approach to identifying cracks in the early stage and on large-scale structures.

\begin{abstract}
Author contributions: Conceptualization, GRG and ZIP; methodology, CT; software, CR, CS and $\mathrm{CT}$; validation, NG and CS; formal analysis MA; investigation, GRG and CT; writing - original draft preparation, MA; writing - review and editing, NG.; visualization, CT; supervision, GRG. All authors have read and agreed to the published version of the manuscript.
\end{abstract}

Funding: This research received financial support through the project" Entrepreneurship for innovation through doctoral and postdoctoral research ": POCU / 380/6/13/123866, a project cofinanced by the European Social Fund through the Operational Program Human Capital 2014-2020.

Data Availability Statement: The data presented in this study are openly available in Mendeley at DOI:10.17632/db94d5ccr6.1 and DOI:10.17632/dn4pxx6b3m.1.

Conflicts of Interest: The authors declare no conflict of interest.

\section{References}

1. Doebling, S.W.; Farrar, C.; Prime, M.B. A Summary Review of Vibration-Based Damage Identification Methods. The Shock and Vibration Digest 1988, 30(2), 91-105. DOI: 10.1177/058310249803000201

2. Radzienski, M.; Krawczuk, M.; Palacz, M. Improvement of damage detection methods based on experimental modal parameters. Mech. Syst. Signal Process. 2011, 25, 2169-2190. DOI:10.1016/j.ymssp.2011.01.007

3. Fan, W.; Qiao, P.Z. Vibration-based damage identification methods: A review and comparative study. Struct. Health Monit. 2011, 10, 83-111. DOI:10.1098/rsta.2000.0717

4. Yang, Y.; Zhang, Y.; Tan, X. Review on Vibration-Based Structural Health Monitoring Techniques and Technical Codes. Symmetry 2021, 13, 1998. DOI:10.3390/sym13111998

5. Gillich, G.R.; Furdui, H.; Wahab, M.A.; Korka, Z.I. A robust damage detection method based on multi-modal analysis in variable temperature conditions. Mech. Syst. Signal Process. 2019, 115, 361-379. doi:10.1016/j.ymssp.2018.05.037

6. Cheng, L; Cigada, A. An analytical perspective about structural damage identification based on transmissibility function. Struct. Health Monit. 2020, 19(1), 142-155. DOI:10.1177/1475921719838079

7. Pacheco-Chérrez, J.; Cárdenas, D.; Probst, O. Experimental Detection and Measurement of Crack-Type Damage Features in Composite Thin-Wall Beams Using Modal Analysis. Sensors 2021, 21, 8102. DOI:10.3390/s21238102 
8. Ravi, J.T.; Nidhan, S.; Muthu, N.; Maiti, S.K. Analytical and experimental studies on detection of longitudinal, L and inverted T cracks in isotropic and bi-material beams based on changes in natural frequencies, Mech. Syst. Signal Process. 2018, 101(15), 6796. DOI: 10.1016/j.ymssp.2017.08.025

9. Bao, A.; Gulasey, M.; Guillaume, C.; Levitova, N.; Moraes, A.; Satter, C. Structural Capacity Analysis of Corroded Steel Girder Bridges. In Proceedings of the 3rd International Conference on Civil, Structural and Transportation Engineering, Niagara Falls, ON, Canada, 10-12 June 2018. DOI:10.11159/iccste18.118

10. Saeedi, K.; Bhat B.R. Clustered Natural Frequencies in Multi-Span Beams with Constrained Characteristic Functions. Shock and Vibration 2011, 18(5), 697-707. DOI: 10.3233/SAV-2010-0592

11. ZI Praisach, N Gillich, I Negru I. Natural Frequency Changes of Euler-Bernoulli Continuous Beams with Two Spans due to Crack Occurrence. Romanian Journal of Acoustics and Vibration 2014, 11 (2), 80-83.

12. Zhang, Y.; Xie, Q.; Li, G.; Liu, Y. Multi-Damage Identification of Multi-Span Bridges Based on Influence Lines. Coatings 2021, 11, 905. DOI:10.3390/coatings11080905

13. Zhou, Y.L.; Abdel Wahab, M. Damage detection using vibration data and dynamic transmissibility ensemble with autoassociative neural network. Mechanika 2017, 23(5), 688-95. DOI:10.5755/j01.mech.23.5.15339

14. Kim, B.; Kim, C.; Ha, S.-H. Multiple Damage Detection of an Offshore Helideck through the Two-Step Artificial Neural Network Based on the Limited Mode Shape Data. Sensors 2021, 21, 7357. DOI:10.3390/s21217357

15. Elshafey, A.A.; Dawood, N.; Marzouk, H.; Haddara, M. Crack width in concrete using artificial neural networks. Eng Struct. 2013, 1(52), 676-86.

16. Zhou, Q.; Ning, Y.; Zhou, Q.; Luo, L.; Lei, J. Structural damage detection method based on random forests and data fusion. Struct. Health Monit. 2013, 12(1), 48-58. DOI:10.1177/1475921712464572

17. Guo, X.; Hao, P. Using a Random Forest Model to Predict the Location of Potential Damage on Asphalt Pavement. Appl. Sci. 2021, 11, 10396. DOI:10.3390/app112110396

18. Huang, D.; Hu, D.; He J.; Xiong, Y. Structure Damage Detection Based on Ensemble Learning. In Proceedings of the 9th International Conference on Mechanical and Aerospace Engineering (ICMAE), Budapest, Hungary, 10-13 July 2018. DOI:10.1109/ICMAE.2018.8467650.

19. Tran-Ngoc, H.; Khatir, S.; De Roeck, G.; Bui-Tien, T.; Abdel Wahab, M. An efficient artificial neural network for damage detection in bridges and beam-like structures by improving training parameters using cuckoo search algorithm. Eng. Struct. 2019, 199, 109637. DOI: 10.1016/j.engstruct.2019.109637

20. Senthilkumar, M.; Manikanta Reddy, S.; Sreekanth, T.G. Dynamic Study and Detection of Edge Crack in Composite Laminates Using Vibration Parameters. Trans. Indian Inst. Met. 2021, 74. DOI:10.1007/s12666-021-02419-y

21. Gillich, G.R.; Praisach, Z.I. Modal identification and damage detection in beam-like structures using the power spectrum and time-frequency analysis. Signal Processing 2014, 96, 29-44. DOI:10.1016/j.sigpro.2013.04.027

22. Gillich, G.R.; Aman, A.T.; Wahab, M.A.; Tufisi C. Detection of Multiple Cracks Using an Energy Method Applied to the Concept of Equivalent Healthy Beam. Proceedings of the 13th International Conference on Damage Assessment of ...

23. Gillich, G.R.; Tufoi, M.; Korka, Z.I.; Stanciu, E.; Petrica, A. The relations between deflection, stored energy and natural frequencies, with application in damage detection. Romanian Journal of Acoustics and Vibration 2016, 13(2), 87-93.

24. Gillich, G.R.; Praisach Z.I. Robust method to identify damages in beams based on frequency shift analysis. Health Monitoring of Structural and Biological Systems 2012 8348, 83481D

25. Gillich, N.; Tufisi, C.; Vasile, O.; Gillich, GR. Statistical Method for Damage Severity and Frequency Drop Estimation for a Cracked Beam using Static Test Data. Romanian Journal of Acoustics and Vibration 2019, 16(1), 47-51.

26. Gillich, G.R.; Maia, N.M.M.; Wahab, M.A.; Tufisi, C.; Korka, Z.I.; Gillich, N.; Pop, M.V. Damage Detection on a Beam with Multiple Cracks: A Simplified Method Based on Relative Frequency Shifts. Sensors 2021, 21(15), 5215. DOI:10.3390/s21155215

27. Negru, I.; Praisach, Z.I.; Gillich, G.R.; Vasile, O. About the Neutral Axis Distortion due to Cracks and its Influence upon the Beams Natural Frequencies. Romanian Journal of Acoustics and Vibration 2012, 12(1), 35-38.

28. [dataset] Tufisi, C.; Gillich, G.R. 2021. Training data for cantilever beam with transverse cracks; Mendeley Data; V1; DOI:10.17632/db94d5ccr6.1

29. Marsland, S. Machine learning: an algorithmic perspective. Chapman and Hall/CRC, 2011.

30. Breiman, L. Random forests. Machine learning 2001, 45(1), 5-32. DOI:10.1023/A:1010933404324

31. Probst, P.; Boulesteix, A.L. To tune or not to tune the number of trees in random forest. J. Mach. Learn. Res. 2017, 18(1), 66736690. https://arxiv.org/pdf/1705.05654.pdf

32. Abiodun, O.I.; Jantan, A.; Omolara, A.E.; Dada, K.V.; Mohamed, N.A.; Arshad, H. State-of-the-art in artificial neural network applications: A survey. Heliyon 2018, 4(11), e00938. DOI:10.1016/j.heliyon.2018.e00938

33. [dataset] Tufisi, C. 2021. FEM simulation results; Mendeley Data; V1; DOI:10.17632/dn4pxx6b3m.1.

34. Mituletu, I.C.; Gillich, G.R.; Maia, N.M.M. A method for an accurate estimation of natural frequencies using swept-sine acoustic excitation. Mechanical Systems and Signal Processing 2019, 116, 693-709. DOI:10.1016/j.ymssp.2018.07.018

35. Nedelcu, D; Gillich, G.R. A structural health monitoring Python code to detect small changes in frequencies. Mechanical Systems and Signal Processing 2021, 147, 107087. DOI:10.1016/j.ymssp.2020.107087

36. [dataset] Gillich, G.R.; Nedelcu, D. Data for: PyFEST - a Python code for accurate frequency estimation; Mendeley Data; V1; DOI:10.17632/g6tc23z48p.1 